\title{
Concept for a Gossamer solar power array using thin-film photovoltaics
}

\author{
Tom Sproewitz ${ }^{1}$ (1) Udayan Banik ${ }^{3}$. Jan-Thimo Grundmann ${ }^{2} \cdot$ Frederik Haack $^{3} \cdot$ Martin Hillebrandt $^{4}$. \\ Hauke Martens ${ }^{2}$. Sebastian Meyer ${ }^{4} \cdot$ Siebo Reershemius ${ }^{2} \cdot$ Nies Reininghaus $^{3} \cdot$ Kaname Sasaki $^{2} \cdot$ Patric Seefeldt $^{2}$. \\ Oleg Sergeev ${ }^{3} \cdot$ Peter Spietz $^{2} \cdot$ Maciej Sznajder $^{2} \cdot$ Norbert Toth $^{2} \cdot$ Martin Vehse $^{3} \cdot$ Torben Wippermann $^{2}$. \\ Martin E. Zander ${ }^{4}$
}

Received: 12 July 2018 / Revised: 25 July 2019 / Accepted: 3 September 2019 / Published online: 17 September 2019 (c) The Author(s) 2019

\begin{abstract}
In recent years, the German Aerospace Center (DLR) developed Gossamer deployment systems in different projects. As power requirements of spacecraft are getting more and more demanding, DLR recently focused on the development of new deployable photovoltaic (PV) technologies that are suitable for generating 10's of $\mathrm{kW}$ per array. Possible space applications that may also require high power supply are missions using electric propulsion such as interplanetary missions, placing of geostationary (GEO) satellites in their orbit or even more future oriented as space tugs or lightweight power generation on extra-terrestrial infrastructures. The paper gives an overview about a feasibility study for flexible solar arrays based on new thin-film photovoltaics. It is expected that the combination of new thin-film PV technologies, e.g., copper indium gallium selenide (CIGS) cells or gallium-arsenide (GaAs) cells, together with Gossamer deployment technologies, could significantly increase the power availability for spacecraft. Based on a requirement, analysis system concepts were evaluated. A focus is on the potential of CIGS PV combined with a two-dimensional deployment of the array and DLR's coilable carbon fibrereinforced plastic (CFRP) booms. Therefore, a concept based on crossed booms with a foldable PV membrane is considered as baseline for further developments. The array consists of rectangular PV generators that are interconnected by flexible printed circuit board (PCB) harness. By a double-folding technique, these generators are laid on top of each other in such that the membrane can be extracted from its stowing box during the deployment in a controlled manner. Considering constantly increasing efficiencies of the CIGS PV combined with Gossamer structures, there is clear potential of reaching a very high specific power value exceeding that of conventional PV systems. Furthermore, the CIGS PV appears to be more radiation resistant and has already reached more than $21 \%$ efficiency in laboratories. Such efficiencies are expected to be achieved in the near future in a standard manufacturing process. However, flexible, thin-film GaAs cells are also subject of consideration within GoSolAr. With this prospect, DLR's research has the goal to develop a Gossamer Solar Array (GoSolAr) to exploit the described potential.
\end{abstract}

Keywords Solar array $\cdot$ Membrane structure $\cdot$ Thinfilm photovoltaic $\cdot$ Deployable structure $\cdot$ Deployment system

Tom Sproewitz

tom.sproewitz@dlr.de

1 German Aerospace Center, Institute of Space Systems, Robert-Hooke-Str. 7, 28359 Bremen, Germany

2 German Aerospace Center, Robert-Hooke-Str. 7, 28359 Bremen, Germany

3 German Aerospace Center, Institute of Networked Energy Systems, Carl-von-Ossietzky-Str. 15, 26129 Oldenburg, Germany

4 German Aerospace Center, Institute of Composite Structures and Adaptive Systems, Lilienthalplatz 7, 38108 Brunswick, Germany

\section{Mission, requirements and scientific output}

The goal of the GoSolAr activity is to develop a technology for large PV arrays based on a two-dimensional deployment combined with flexible PV and to demonstrate the functionality in-orbit.

GoSolAr is intended to be a deployable PV array system of the size of $5 \mathrm{~m} \times 5 \mathrm{~m}$ which shall be used to demonstrate necessary technologies for future array sizes of about $20 \mathrm{~m} \times 20 \mathrm{~m}$, including scalability. The required array size 


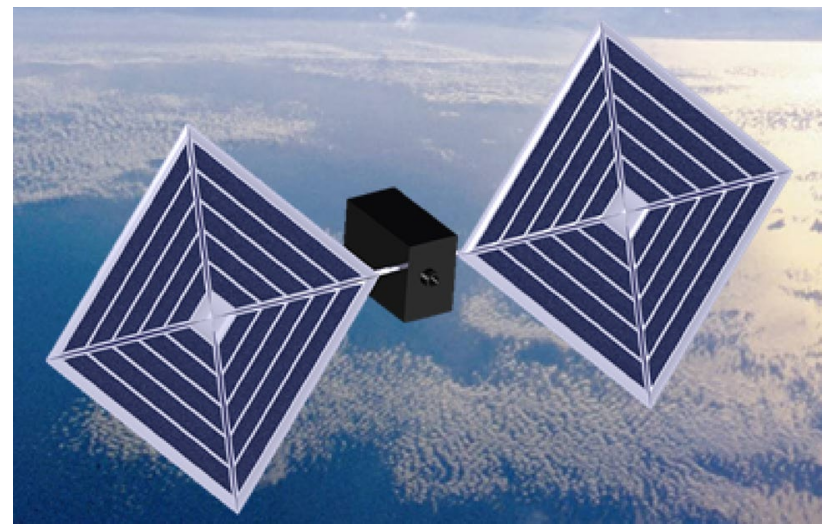

Fig. 1 Artists view of high power demand spacecraft with Gossamer photovoltaic arrays

for a given power output depends on the efficiency of the available thin-film PV. Figure 1 shows a concept of a high power demand spacecraft configuration with electric propulsion. Figure 2 shows the GoSolAr demonstrator combined with the future DLR small satellite bus in stowed and deployed configuration [1].

GoSolAr will consist of a so-called PV blanket which is deployed and supported by deployable CFRP booms and their deployment units. The PV blanket consists of PV generators, the harness and connecting elements. Customized avionic hardware and software is designed for operation and PV characterization.

GoSolAr will be designed as an experiment. Therefore, it has a specific electrical layout including PV characterization electronics and a limited amount of applied PV. Two times $140 \mathrm{~W}$ is envisaged for the experimental PV to enable small satellite bus powering for demonstration purposes. The remaining area of the array will be equipped with mass and stiffness representative dummies PV.
In contrast to many membrane deployment technologies, the deployment shall be conducted in a controlled manner, meaning no free floating membrane area at any time. Both fragile elements, membrane and deployable booms shall be robust against a higher number of deployments.

The outcome of the activity will demonstrate the:

- Applicability of the overall, deployable Gossamer thinfilm PV array system.

- Potential of realizing decisively higher power/mass ratios compared to conventional PV array technologies.

- Suitability of the thin-film PV for space applications.

- Potential of the scalability of the main technologies and their combined use as a large system.

It is envisaged to perform a full on-ground qualification test campaign by means of a qualification model before establishing a flight unit. The planned in-orbit verification shall prove the safe two-dimensional deployment of the CFRP booms and the flexible $5 \mathrm{~m} \times 5 \mathrm{~m}$ array, which is partially equipped with operational thin-film PV and all corresponding mechanisms. It shall show the possibility of a safe operation of the mission satellite and the deployable PV array system during and after deployment as well as during power generation. In this context, the characterization of the dynamics of the deployment is of particular interest.

Furthermore, the space qualification of CIGS technology is a main goal even though GaAs PV technology is also examined within this activity. The investigation of the degradation under space conditions will be a key insight for future projects. The prospect of space qualification of CIGS solar cells has met great anticipation and willingness to contribute by most of the known manufactures.
Fig. 2 Artists view of GoSolAr on S2TEP (left) and deployed GoSolAr demonstrator array (right)
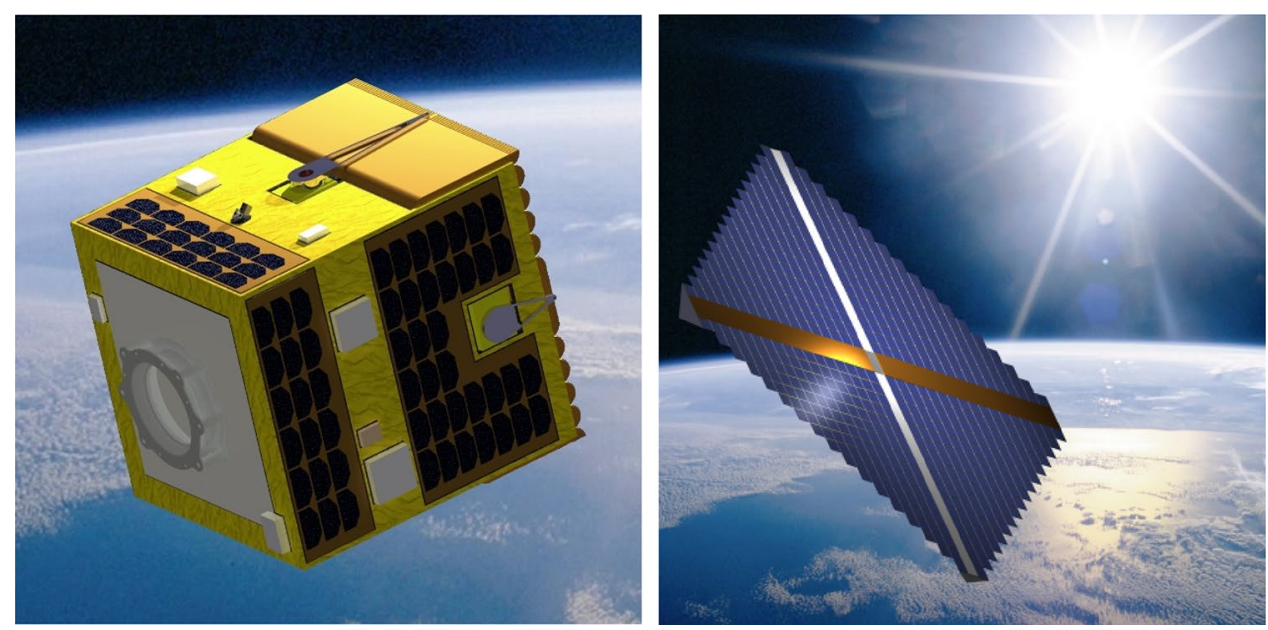


\section{Overall accommodation}

Figure 3 gives an overview of the mechanical components of GoSolAr in an early state. This accommodation shows the concept for a sequential unfolding of the PV blanket, which requires two CFRP boom deployment units.

One of the specific challenges for GoSolAr is the need to store the $5 \mathrm{~m} \times 5 \mathrm{~m}$ PV blanket and to allow for deployment in all directions in the blankets plane $(x-y$ plane in Fig. 3). Therefore, all side walls of the outside structure need to secure the PV blanket in its stowed configuration and move out of the blanket plane prior to its deployment. This movement of the side walls will be realized by either folding the walls out of the blanket plane or by attaching the walls to the deployable boom tips and deploying them with the booms. This excludes the side walls from contributing to the main structure to carry mechanical loads. It is required to place this main structure through the centre of the PV blanket along the $z$-axis. All harnesses from the PV blanket and the top cover need to be routed through the centre as well. At the same time, the deployment units need to be placed in the centre directly underneath the PV blanket to reduce bending moments on the booms.

The volume envelope for GoSolAr is related to the dimensions of the small satellite bus and is specified with $(500 \times 500 \times 350) \mathrm{mm}^{3}$. The overall height of the stowed blanket and the deployment units make it necessary to position one deployment unit between both packages of the stowed PV blanket (upper deployment unit in $z$-direction in Fig. 3). This limits the available space for the stowed blanket, deployment unit, structure and harnesses.

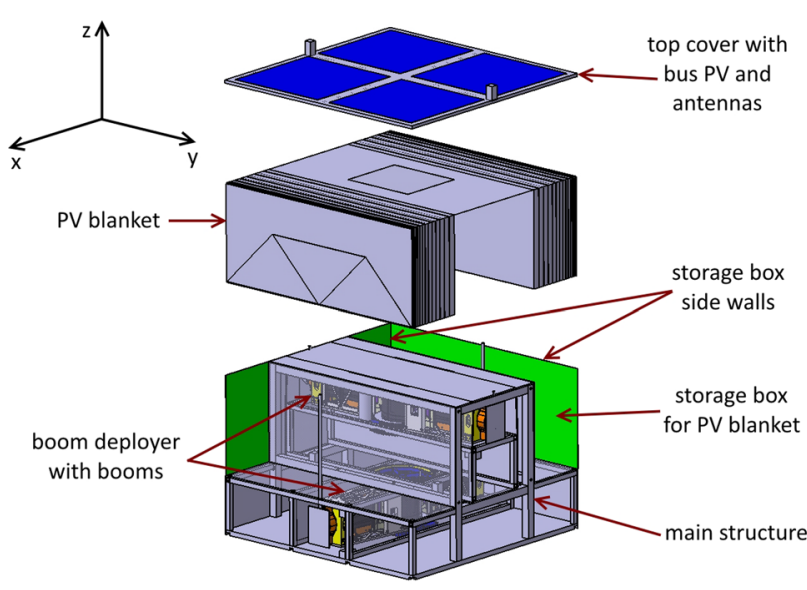

Fig. 3 GoSolAr accommodation concept

\section{Overall electrical layout}

According to GoSolAr's mission definition, the overall electrical layout is focussed on demonstration and characterisation of the new thin-film PV technologies rather than on power output optimisation, as it would be for an operational power generator. Nevertheless, credible demonstration of power generation by temporarily powering the satellite bus is a design driver.

In view of technology demonstration, a single PV generator strategy is followed, where each PV generator is connected to the GoSolAr power conditioning and characterisation electronics individually. This enables maximum flexibility regarding accommodation of PV generators of different technology in different locations on the blanket as well as maximum flexibility for characterisation measurements. With each PV generator having its own power tracks as well as electrical measurement tracks, arbitrary electrical string connections can be defined with correspondingly different output voltages by suitable multiplexing units within GoSolAr's electronics. This maximizes the design independence from the satellite bus battery charging concept in terms of end of charge voltage. At the same time, this is part of the project's mitigation strategy regarding not fully predictable availability of suitable thinfilm PV technology providers. Late changes of PV generator design are thereby possible. Furthermore, electrical characterisation of each PV generator individually as well as of arbitrary combinations is enabled.

It is understood and accepted that by this single PV generator approach, unnecessarily long electrical connections with corresponding losses are introduced.

\subsection{Photovoltaic technology}

Solar cells in extra-terrestrial applications are dominated by the GaAs-(III-V) multijunction technology. Those cells are manufactured in very small numbers expressly for space applications. Manufacturers are, e.g., Azur Space, CESI and MicroLink. Their second purpose-usage in concentrator photovoltaics (CPV)—has seen a rapid decline. Since silicon PV prices dropped so low, CPV is not cost efficient any more. Those two facts are the reason for the high prices for those cells of approximately $200 € / \mathrm{Wp}$ (Watt Peak) or less depending on quantity and quality. Although the III-V multijunction solar cells have become very thin (below $100 \mu \mathrm{m}$ thickness), they are only bendable in one direction simultaneously and are vulnerable to mechanical impacts. For protection against particle irradiation, they are often sandwiched with a cover glass losing weight and flexibility benefits. The bare cells are 
very lightweight because of their thickness: $80-\mu \mathrm{m}$-thick cells from Azur Space for example weigh less than $1.6 \mathrm{~g} /$ $\mathrm{Wp}[2]$.

There are two main reasons to use III-V multijunction cells despite their high costs. The efficiency is with $\sim 30 \%$ higher than any other technology, i.e., for $1 \mathrm{Wp}$ power generation only $32 \mathrm{~cm}^{2}$ have to be allocated. The second reason is the stability. III-V multijunction cells are certified to withstand extra-terrestrial radiation without degradation. From BOL to EOL $\left(10^{15} 1 \mathrm{MeV} \mathrm{e}^{-} / \mathrm{cm}^{2}\right)$, they show only $10 \%$ loss in efficiency [2].

In the GoSolAr activity, it is planned to use CIGS thinfilm solar cells on polyamide substrates. They are truly flexible down to a $25 \mathrm{~mm}$ roll radius (see Fig. 4), very lightweight $(1.3 \mathrm{~g} / \mathrm{Wp})$ and cost only $20 € / \mathrm{Wp}$ [3].

Manufacturers are MiaSolé, Flisom, Globalsolar, Sunplugged and Ascent Solar. The efficiency of CIGS is lower than the III-V multijunction technology. With production efficiencies around $15 \%$ and laboratory efficiencies above $20 \%$ [4] we see the efficiency gap between the two technologies reducing in the future. Especially at AM0 conditions, CIGS PV has less than half of the efficiency of the III-V multijunction cells leading to comparably large areas. But, as no stiff support structures are necessary, the CIGS PV can be competitive with III-V multijunction photovoltaic array systems.

A trend in the commercially available III-V multijunction technology at the moment is to produce thinner, flexible and less expensive cells at the same efficiency level with manufacturers like Alta Devices in the USA. This technology is also of interest for the flexible solar array development and is meanwhile implemented in GoSolAr as one of the potential PV technologies to be demonstrated.

So far, there are limited reliable data for the performance of CIGS under extra-terrestrial radiation. However, the preliminary data suggest very good performance of CIGS solar cell technology under such conditions, even outperforming III-V multijunction technology in terms of degradation behaviour [5], [6].
In LEO, the surface temperature of solar panels can vary from -120 to $+120{ }^{\circ} \mathrm{C}$ due to sunlight irradiation [7]. Thermal emissivity of CIGS cells is generally in the range of $0.17-0.28$ due to the very high far infrared reflectance of the cell $[8,9]$. This results in loss of denominated cell power at elevated temperatures due to its unfavourable radiative heat transfer properties. Having a high emissivity $(\varepsilon)$ coating on the cells allows dissipation of heat but needs to be flexible. Pscherer et al. in co-ordination with DLR demonstrated that a SiOx layer using dip coating technique from polysilazane precursor material has good emissive properties [10]. The $\varepsilon$ of the CIGS stack increases to 0.78 in the infrared range of 2.5-25 $\mu \mathrm{m}$ using a SiOx coating from a Durazane 1800 precursor without losing spectral absorbance. A sufficient bending radius of $20 \mathrm{~mm}$ was achieved with a $2.2 \mu \mathrm{m}$ coating and, therefore, flexibility and adherence was not compromised. Consequently, polysilazane-based $\mathrm{SiOx}$ coatings will be used on the CIGS modules of GoSolAr. The novelty is that the coating can be carried out at low temperature where a post-processing step involves annealing at $200{ }^{\circ} \mathrm{C}$. This coating will also serve as a barrier to low-energy protons and AtOx etching in space.

The PV generators will be sized approximately $200 \times 200$ $\mathrm{mm}$. Since the cells on each generator are series interconnected by laser scribing [10], they can be tailored to fit the battery voltage of the system they are providing. Depending on the manufacturer of the bare PV, it might be necessary to connect two PV generators in series to reach the desired voltage. Thermal and mechanical contact to the harness will be achieved by glueing the generator on top of the harness. Electrical contacts will be done by weaving loops of the electrical harness over the generator and glueing it with conducting glue to the front and back contact of the PV generator.

For characterization of the solar cells performance and to study the degradation of the cells over time, the open circuit voltage of the cells will be continuously observed combined with a current measurement. This will be realized by a switchable network of customized low resistance shunt
Fig. 4 Flexible CIGS modules; Left: Flisom; Right: Ascent Solar
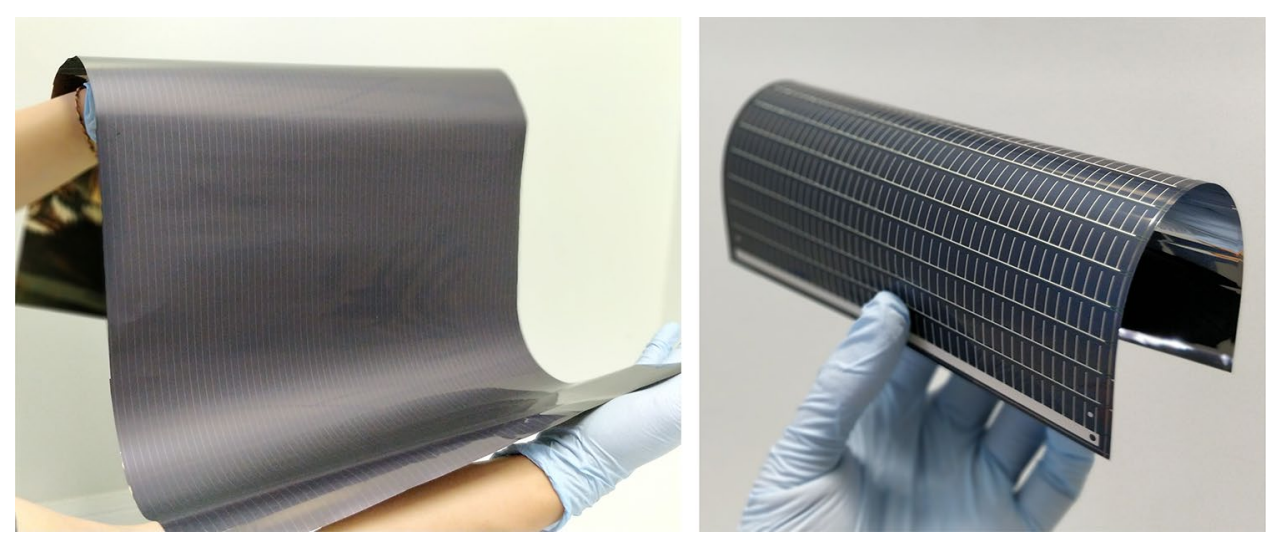
resistors to derive proper voltage and current characteristics. Additionally the single cell temperature, particle count and irradiance shall be continuously monitored. By combining the data, ageing or self-healing processes of the cells can be observed during mission time.

\subsection{Photovoltaic blanket}

The photovoltaic blanket which will be deployed in two dimensions consists of:

- PV generators,

- harness to connect all generators to the power conditioning and characterisation electronics,

- membrane to interconnect PV generators and harness as well as to connect the PV blanket to the deployable booms, which span the PV blanket over the full area.

The mechanical design of the PV blanket has to take into account demanding requirements, such as:

- low mass budget of less than $8 \mathrm{~kg}$ for the $25 \mathrm{~m}^{2}$ blanket,

- small stowing volume inside the spacecraft,

- repeatability of the deployment of at least 10 times under Earth conditions.

To fulfil these requirements, the membrane has to be thin, to allow for small bending radii, and lightweight. At the same time, it has to be sturdy enough to avoid damages. Additionally, the PV blanket has to cope with the severe thermal conditions caused by the thermal properties of non-operational PV generators for the worst case. To avoid destructively high temperatures while the PV blanket is illuminated by the Sun, the desired optical properties for the backside material are high emissivity and low absorptivity. Especially high emissivity is important to accomplish sufficient heat rejection. Considering these thermal requirements, the following options for thermo-optical design are being investigated:

- A high emissivity SiOx coating on PV.

- A supporting layer made of black polyimide film backside (high emissivity, but also high solar absorptivity).

- A high emissivity SiOx coating on the backside of the PV blanket.

Other possibilities were also examined, but had their limitations in the mechanical use or in the impact on the mass budget.

Stowing concepts shall ensure that the deployment is repeatable, controlled and robust. Therefore, different folding patterns are under investigation, which are reliable and simple enough to avoid complicated mechanisms. The folding pattern also has implications on other parts of GoSolAr, like the number of required CRFP boom deployment units (e.g., a simultaneous deployment in both directions or a twophase deployment as shown in Fig. 5).

Additional features on the PV blanket are considered to limit the uncontrolled movement of the blanket during the deployment. The goal is to have a stable stack of the folded blanket in a force-free environment, which will only unfold, when the boom deployment units pull on the blanket.

The PV generators are arranged in columns parallel to the diagonals of the PV blanket. Each generator is connected to one of several flexprint harnesses that are routed along those columns towards one diagonal where the main harness is located (see Fig. 6). There all column row harnesses are brought together. Slack in the harnesses between each PV generator, which is required to fold the blanket and still fulfil the minimal bending radius of the harness, is again dependent on the folding pattern.

The electrical layout of the harness is such that standard Flex PCB manufacturing technologies can be utilised. Furthermore, the layout enables identical interfaces to all
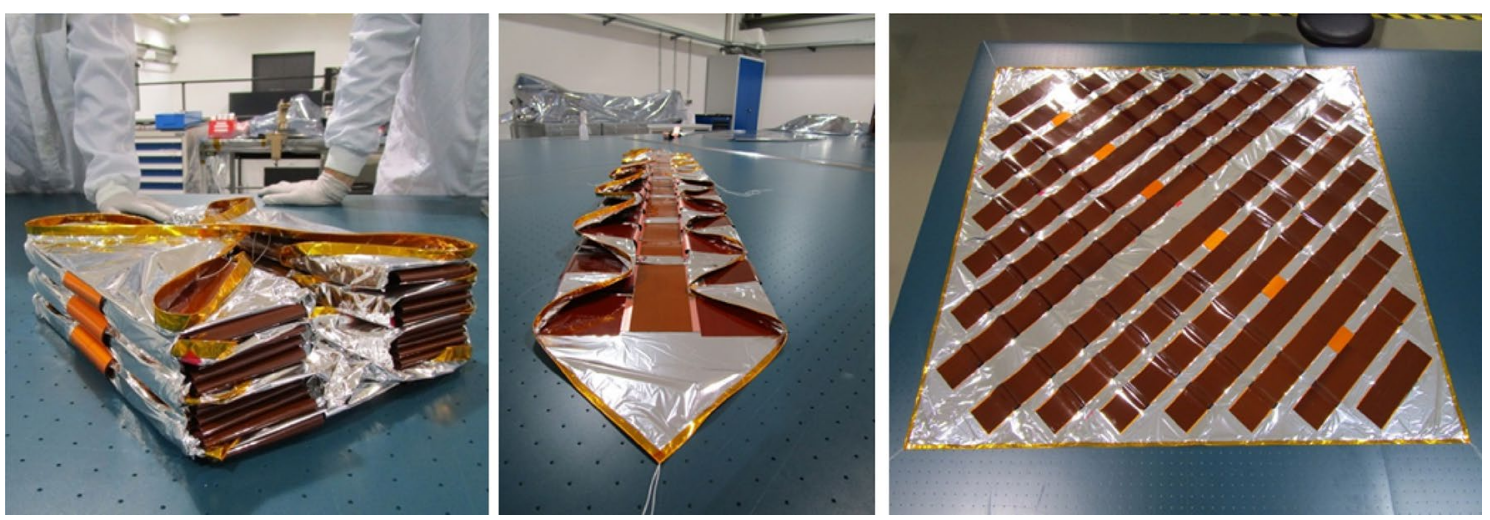

Fig. 5 Membrane two-phase deployment scheme: left: packed, mid: first direction deployed, right: second direction deployed 


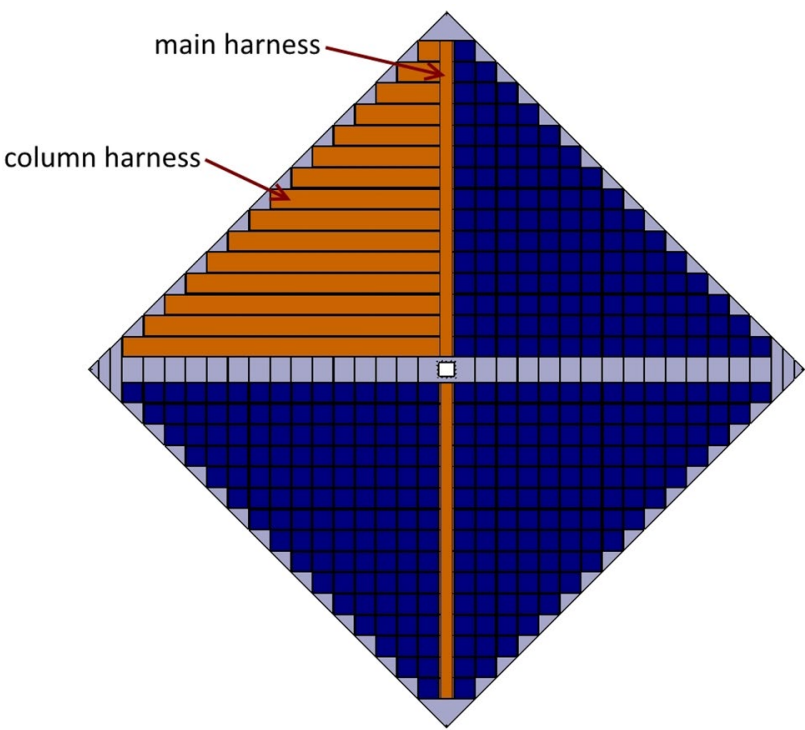

Fig. 6 Photovoltaic blanket with PV generators and a principle illustration of column harness and main harness

PV generators, i.e., same electrical-geometrical layout of all PV generators. To avoid hot spots caused by electrical losses in harness power tracks as well as to achieve thermally homogeneous conditions in the area below the PV generators, the harness will cover the full width of the PV generators. Power tracks will be "smeared out" across the full available width, while for mass optimisation they are at the same time fashioned in a latticed way, similar to electrical screening areas in standard PCB layouts as shown in Fig. 7.

While achieving homogeneous conditions below each individual PV generator, it is understood that mechanical and thermal properties are different below different generators as well as between different rows due to this harness approach. This illustrates a specific aspect of this approach, where electrical, mechanical, and thermal aspects are highly integrated within a few tens of millimetres of thickness of the GoSolAr blanket.

Effective cross-sections of the harness power tracks are defined based on initial standard derating approaches followed by dedicated thermal modelling of the full blanket [12], [13]. This is cross-checked by W/kg-optimisation, with harness cross-section (driving harness $\mathrm{Cu}$ mass) as optimisation variable.

The present approach does not consider a compensation of differences in resistance of electrical tracks due to different connection length by length proportional cross-section increase. Such compensation would facilitate simpler and more efficient power-conditioning electronics. However, it is not excluded that this might be an option to be considered for large systems.

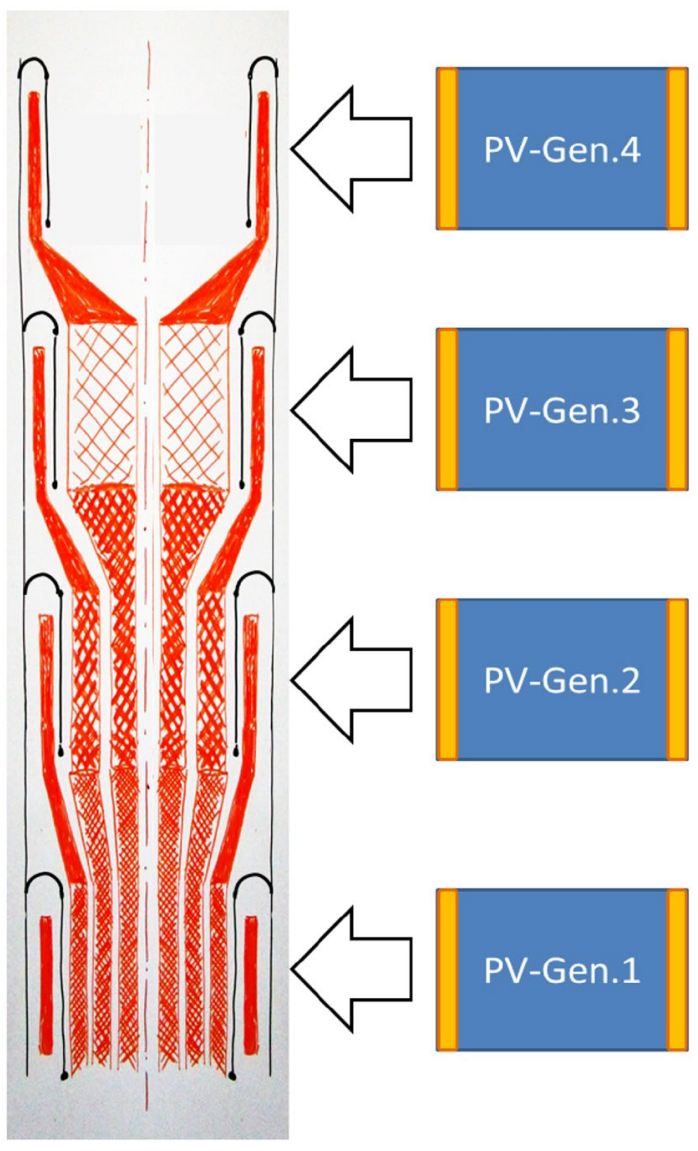

Fig. 7 Latticed column harness which contains 4 PV generators. Only power tracks for connecting the PV generators are shown

\section{Deployable boom technology}

The PV blanket of the GoSolAr satellite is structurally supported by four of DLR's reelable double-omega booms as shown in see Fig. 8 [14-16]. This boom type possesses a closed, tubular cross-section that consists of two

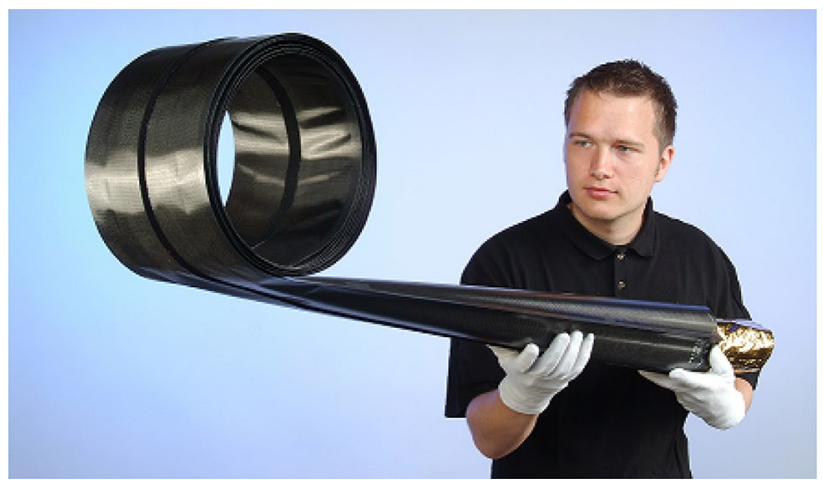

Fig. 8 DLR's tubular, reelable boom in partially deployed state 


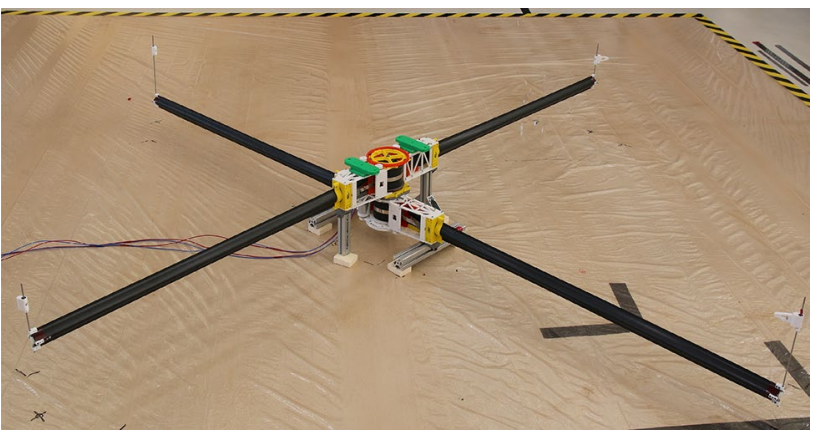

Fig. 9 Breadboard model of GoSolAr's deployable support structure consisting of four cross-wise aligned booms with two central deployment units

omega-shaped half-shells which are joined at their flanges. The shells are made of a thin-walled carbon fibre composite material which features both high stiffness and high elastic strain. These design characteristics enable the tubular boom to be compressed to a flat band and reeled up afterwards on a drum for compact stowage.

The four booms are running in a cross-configuration underneath the rectangular PV blanket along its diagonals. The booms are attached to the four blanket corners at their tips by vertical poles to overcome the offset between booms and blanket mid-plane. In the centre of the array, two deployment units are located (see Fig. 9). Each unit contains a set of two opposing booms on a single drum which are simultaneously deployed. The deployment units thereby also drive the unfolding of the PV blanket. Two separate deployment units are necessary to enable the unfolding of the PV blanket in the two spatial dimensions in separate steps.

The primary design requirements of the booms are a high bending and compression strength as well as a small stowed volume. The strength requirement results from the offsets between blanket and boom plane which leads to bending moments introduced at the boom tips. The primary sources of loading during deployment are the forces required to achieve release of the retaining clips which keep the blanket in its folded state.

The design of the boom is strongly linked to the design of the deployment units (see Fig. 10). The booms possess a transition zone between the fully flattened and fully opened state. This transition zone leads to reduced mechanical properties due to the reduced cross-sectional dimensions and requires additional support from the outside by the surrounding deployment unit. However, the transition zone cannot be contained in full length especially as a low stowed volume is a key design driver. Thereby the overall mechanical properties are a result of the shape and length of the transition zone and the length and quality of the support given by the surrounding deployment unit. In consequence, a main design focus of the deployment system is a short and

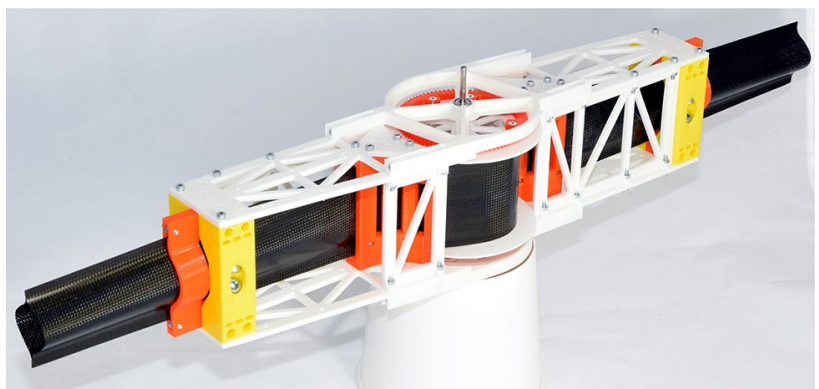

Fig. 10 Breadboard model of a single deployment unit with two opposing booms reeled on a central drum

well-supported transition zone that is tailored to maximize the overall strength and stiffness.

Each deployment unit (see Fig. 10) contains two opposing booms that are reeled on one central drum. The booms are deployed by an electric motor that applies the deployment force to the booms through co-coiled steel belts. The belts are reeled on a separate spool which is attached to the motor. By reeling the belt on the separate spool, the booms are pulled off the drum. Adjacent to the central drum are the truss-like structures with shells in the form of the opening boom at their ends. These shells contain the boom and provide the required additional support to the transition zone to enhance the overall strength and stiffness.

\section{Membrane and boom-combined breadboard deployment testing}

To verify the main concept of the PV blanket in a very early stage, a breadboard model was tested. A $1 \mathrm{~m} \mathrm{x} 1 \mathrm{~m}$ membrane demonstrator with PV mass dummies was deployed from its storage box by two deployment units extracting two booms each (see Figs. 12, 13). As connecting hinge between the PV generators, an elastically deformed flex PCB type A was foreseen (see Fig. 11). This demonstrator test helped to understand the behaviour of the blanket during deployment. The combination of different layers of base foil, harnesses and generators distributed over the PV blanket is otherwise difficult to simulate (both in hardware and software).

One additional combined deployment test using a membrane with the flex PCB hinge Type D (see Fig. 11) has also already been performed and showed an advantageous behaviour compared to the one described hereafter. However, the data could not yet be analysed to allow for a publishing.

\subsection{Breadboard composition and test setup}

The blanket breadboard had the setup of column and main harnesses as shown in Fig. 6. Hinge option A between the 


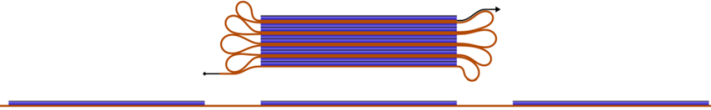

A: Elastically deformed flex PCB hinge concept

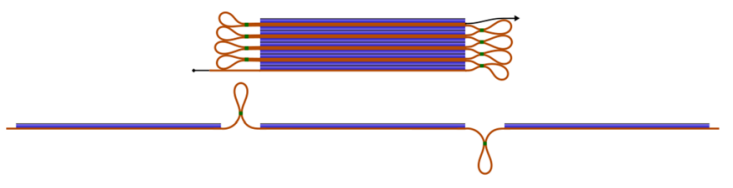

D: Elastically closed loop flex PCB hinge concept

Fig. 11 Flex PCB hinge concepts for interconnection of PV generators

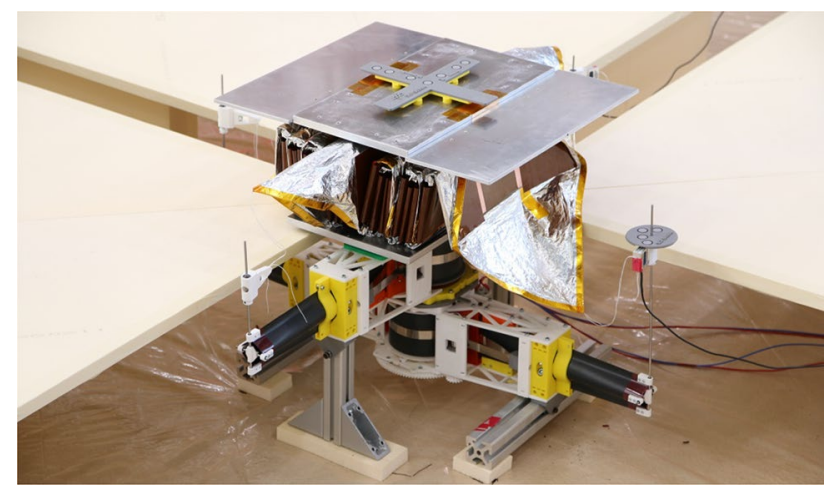

Fig. 12 Deployment test breadboard setup (2 boom deployment units with 2 booms each and one PV blanket stowed in storage box)

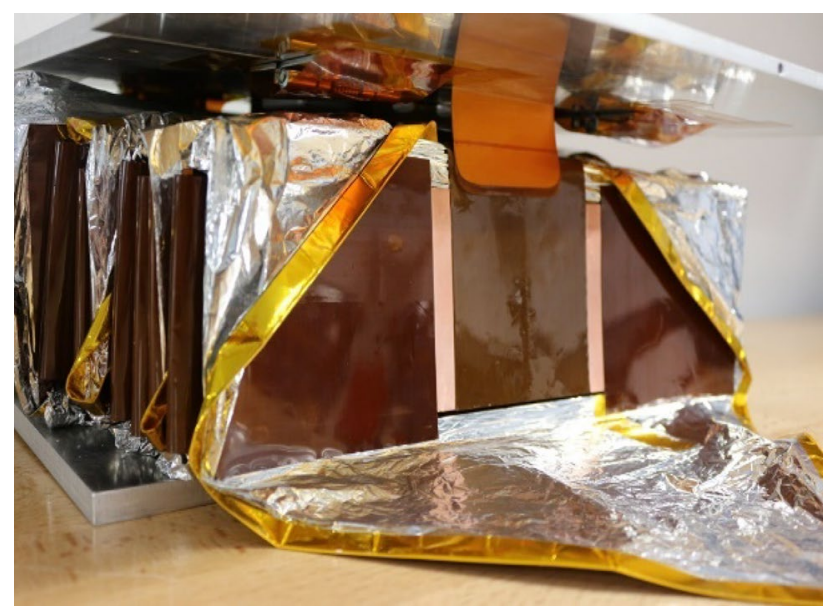

Fig. 13 Storage box with PV blanket dummy and lamella leave

generators was foreseen as flex PCB hinges for main and column harnesses. Hence, the hinges were bent elastically resulting in a blanket, which tends to unfold on its own in a force-free environment. The generators and harnesses were glued to a $12-\mu \mathrm{m}$-thin aluminized polyimide foil.

The deployment test campaign was planned together with a functional breadboard of booms and boom deployment units as shown in Fig. 12. The test setup consisted of the two deployment units at the bottom and perpendicular to each other. The storage box with the blanket was mounted on top. To compensate the weight force of the blanket, four triangular-shaped deployment tables were positioned around the deployment units and storage box to build a square with gaps along the diagonals where the booms are located. The height of the deployment tables was chosen such that the blanket rests on the blocks during and after deployment and hence compensates the membrane mass.

Lamella leaves to prevent spontaneous unfolding of the PV blanket because the elastically deformed harnesses between the generators were foreseen (see Fig. 13). For the second deployment, direction friction clips were used to keep the several blanket layers stowed.

The two-phase deployment scheme, as described above, is realized with subsequently deployed directions, as illustrated in Fig. 14 top and bottom.

Two force sensors were mounted at the boom tip-blanketinterfaces to acquire the forces needed to pull the blanket folds out of the storage box, forces needed to tauten the blanket and stretch the folds, and consequently to detect the acting forces at the boom tips. Force Sensor 1 was installed in the first deployment direction and Force Sensor 2 in the second direction. Both sensors were recording during the deployment in both directions. Additionally, optical targets (see grey circular plate on boom tip in Fig. 12) were placed at each point of load measurement to track translational as well as rotational displacements of the boom tips with a photogrammetry system. However, within the here presented preliminary tests, only the two deployment directions are analysed.

\subsection{Test results and conclusions}

The acquired data are analysed in two ways: in force-overtime as depicted in Fig. 15 and in force-over-deployed boom length as shown in Fig. 16. The force-over-time behaviour is plotted such that the subsequently performed deployment phases in the two directions are shown, thus resembling the complete deployment process.

The force measured by sensor 1 in direction 1 shows small peaks between $1 \mathrm{~N}$ and $2 \mathrm{~N}$ (see Fig. 15). This is the required force to pull the blanket passes the lamella leaves. During the last $10 \%$ of the distance, the tauting of the blanket started, which resulted in a maximum force of approx. $8 \mathrm{~N}$ for deployed boom length of $900 \mathrm{~mm}$. The load at the 


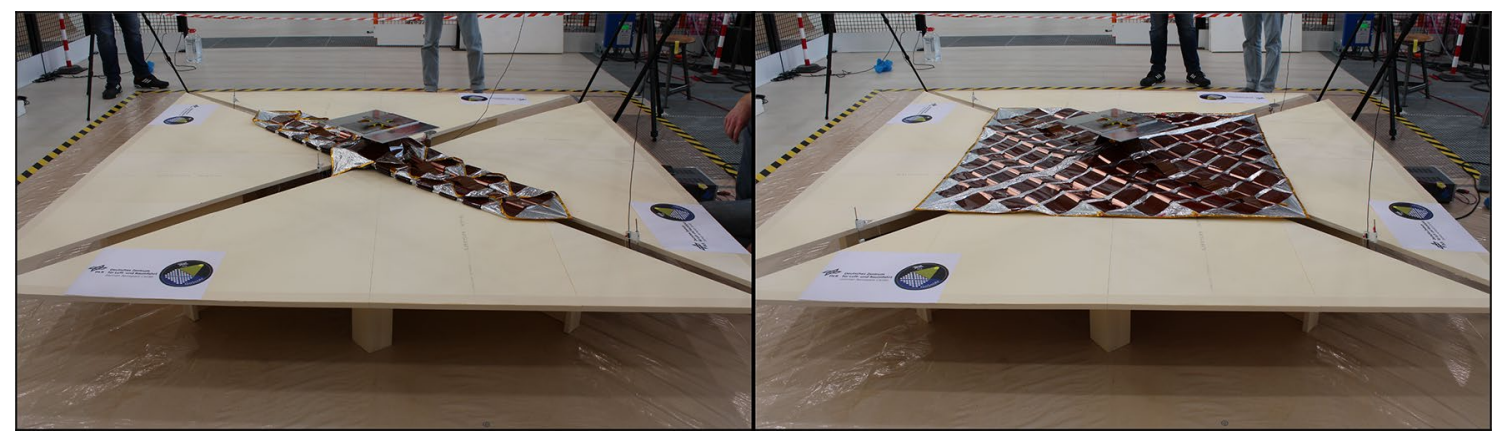

Fig. 14 Left: demonstrator deployment first direction deployed; right: demonstrator fully deployed

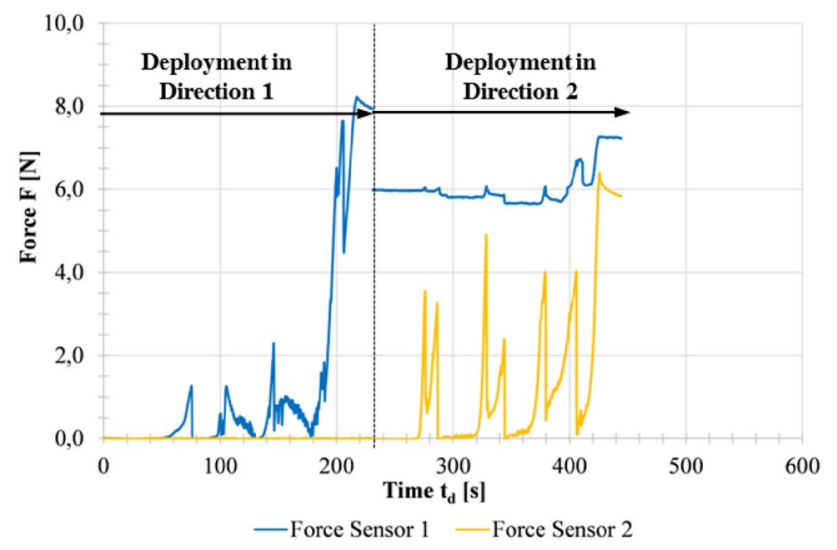

Fig. 15 Test results-force-over-time

passive (still stowed) boom tip (see yellow curve, force sensor 2 in Fig. 15) remains at a value around zero.

In the second deployment direction (see Fig. 15), perpendicular to direction 1, the sensor 2 was recording a different force profile. Now the boom with force sensor 1 (blue curve) will remain passive, since it is already deployed, and force sensor 2 (yellow curve) measures the loads introduced into

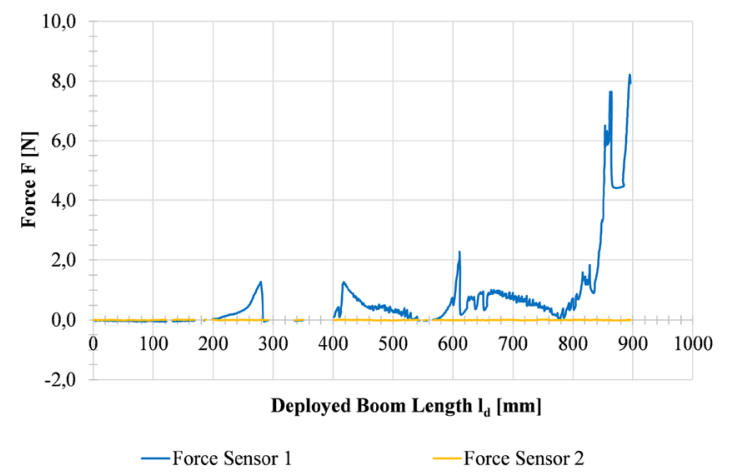

first deployment direction the actively deploying booms. Here again, for the actively deploying boom, frequent peaks in the curve occur every time the friction clips were released. Two peaks with an increasing offset were recorded. This is caused by the arrangement of the friction clips. There were two friction clips per blanket layer and they were positioned at the outmost position for each layer. When a blanket layer was unfolded, only one friction clip was released first. This gave enough available free length of the blanket, that the boom tips could move on for some time without force until the second friction clip was released. As the deployment went on, the current unfolding blanket layer was wider and with it the distance between friction clips.

The observed bending moments acting on the booms, caused by the restraining force of the friction clips, are undesirable. They result in higher stresses for the CFRP booms and can induce a spin on the satellite while in orbit. Therefore, the friction clip concept needs to be revised.

Marking the tautening of the blanket, a steep increase of the curve is clearly visible. The passive booms are experiencing a constant high level of loading while small peaks appear, synchronously to the peaks of the active boom. This indicates that load build ups due to the release of the folds

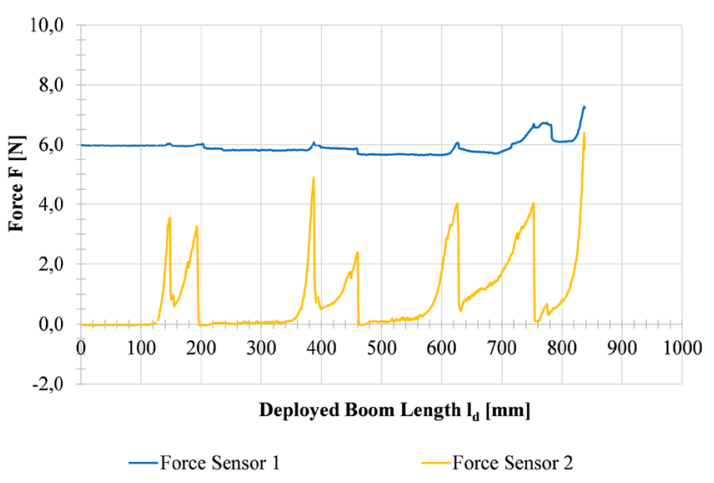

Second deployment direction

Fig. 16 Test results-force-over-deployed boom length 
from the blanket storage box are also detectable at the boom tips of the passive boom. To the end of the deployment process, the active and passive booms show a force increase due to tautening the blanket. After a while of settling, the tension in the blanket releases and forces in both direction balance out at a similar load level. However, due to the stiffness of the blanket folds (dummy flexible PCB), it was not possible to stretch the PV blanket to complete planarity within the threshold load, that prevents the structural system from collapsing.

Showing the same mechanical characteristic in both types of graphs, the force-over-time charts give a good overview of events during the deployment process, being able to link membrane events with boom loading, the forceover-deployed boom length charts give valuable feedback at which boom length a load occurs. This is important due to the fact that with increasing boom length, the leverage arm it represents, as well as the resulting buckling loads increase to a critical level.

\section{Avionics}

As GoSolAr will be designed as a payload, an own electrical bus system is not foreseen. The GoSolAr Command and Data Handling (C\&DH) subsystem has a data interface for telemetry and telecommand and a power interface which shall be controlled from the mother spacecraft bus system. The C\&DH has two main functionalities:

- Solar array deployment control and

- PV characterization.

The C\&DH should be implemented as a cold redundant system. Therefore, the electronics will be composed of:

- 2 Payload Board Computers,

- 2 Payload Power Control Units,

- 2 PV Characterization Electronics,

- 2 Motor drivers for the deployment.

It will be developed either as two independent redundant strings or as a cross-connected redundant payload electronics. The payload PCBs will be mounted in a separated electronic box which will be connected to the mother spacecraft.

\section{Summary and outlook}

The paper gives an overview on the concept for a large, lightweight, deployable Gossamer PV array based on thinfilm PV and deployable CFRP booms that is currently being developed at DLR. It provides a system overview including a description of all main technologies that are brought together in the system. Already in the current design status power/ mass ratios comparable to conventional PV arrays seem feasible to achieve.

Currently, a proper selection of available CIGS PV is ongoing. However, two manufacturers are closely investigated which are Flisom and Ascent Solar. Both have efficiencies around $10 \%$ and both manufacturers give a prospect of efficiency increase in the standard production process in the upcoming months. Beyond CIGS PV, also thin-film triple junction GaAs cells are currently under investigation.

Together with the PV selection, the investigation of concepts to keep the PV within operating temperature ranges, like layering the blanket or to foresee special high emitting coatings, is ongoing. One of the main technical issues is a proper contacting between PV and harness and in between the harness itself which is currently investigated with high priority.

First deployment tests with a demonstrator consisting of a PV blanket dummy and two boom deployment units with two booms each were successfully performed. Hereby deployment forces were monitored. From the data gained, design changes had to be implemented. Especially the stowing of the membrane and the design of the folded harness lines had to be reconsidered to avoid high deployment peak forces and to avoid a spontaneous deployment of the blanket. Deployment tests with adapted technologies were already performed during the preparation of this paper but could not be processed for a presentation.

Open Access This article is distributed under the terms of the Creative Commons Attribution 4.0 International License (http://creativeco mmons.org/licenses/by/4.0/), which permits unrestricted use, distribution, and reproduction in any medium, provided you give appropriate credit to the original author(s) and the source, provide a link to the Creative Commons license, and indicate if changes were made.

\section{References}

1. http://www.dlr.de/irs/en/desktopdefault.aspx/tabid-12525/21846 read-49985/

2. http://www.azurspace.com/images/products/0004148-00-01_DB GBK_80\%C2\%B5m.pdf

3. https://flisom.com/wp-content/uploads/2017/08/Datasheet_ultra -light-CIGS-eFilm-solar-cell-material.pdf

4. Chirilă, A., Reinhard, P., Pianezzi, F., Bloesch, P., Uhl, A.R., Fella, C., Tiwari, A.N.: Potassium-induced surface modification of $\mathrm{Cu}(\mathrm{In}, \mathrm{Ga}) \mathrm{Se}_{2}$ thin films for high-efficiency solar cells. Nat. Mater. 12(12), 1107-1111 (2013). https://doi.org/10.1038/nmat3 789

5. Kawakita, S., Imaizumi, M.: Space demonstration of $\mathrm{Cu}$ (In, Ga) Se 2 solar cells and modules by japanese small satellites. Eur. Photovolt. Solar Energy Conf. Exhibition 31, 1407-1410 (2015)

6. Brunner, S., Zajac, K., Seifart, K., Hartmann, L., Otte, K.: Inflight results of the flexible solar cell panel on tet-1. In: 10th European Space Power Conference, pp 15-17 (2014) 
7. Chen, J., Ding, N., Li, Z., Wang, W.: Organic polymer materials in the space environment. Prog. Aerosp. Sci. 83, 37-56 (2016)

8. Shimazaki, K., Imaizumi, M., Kibe, K.: $\mathrm{SiO} 2$ and $\mathrm{Al}_{2} \mathrm{O}_{3} / \mathrm{SiO}_{2}$ coatings for increasing emissivity of $\mathrm{Cu}(\mathrm{In}, \mathrm{Ga}) \mathrm{Se} 2$ thin-film solar cells for space applications. Thin Solid Films 516(8), 2218-2224 (2008)

9. Günthner, M., Pscherer, M., Kaufmann, C., Motz, G.: High emissivity coatings based on polysilazanes for flexible $\mathrm{Cu}(\mathrm{In}, \mathrm{Ga}) \mathrm{Se} 2$ thin-film solar cells. Sol. Energy Mater. Sol. Cells 123, 97-103 (2014)

10. Pscherer, M., Günthner, M., Kaufmann, C.A., Rahm, A., Motz, G.: Thin-film silazane/alumina high emissivity double layer coatings for flexible $\mathrm{Cu}(\mathrm{In}, \mathrm{Ga}) \mathrm{Se} 2$ solar cells. Sol. Energy Mater. Sol. Cells 132, 296-302 (2015)

11. Westin, P.-O., Zimmermann, U., Edoff, M.: Laser patterning of P2 interconnect via in thin-film CIGS PV modules. Sol. Energy Mater. Sol. Cells 92(10), 1230-1235 (2008). https://doi. org/10.1016/J.SOLMAT.2008.04.015

12. Gsfc, D.G.Cleveland, et al.: GSFC-PPL-19—Goddard Space Flight Center Preferred Parts List PPL-19. Goddard Space Flight Center Parts Branch, Maryland (1989)

13. NASA, K. Sahu et al.: NASA EEE-INST-002-instructions for EEE parts selection, screening, qualification, and derating,
National Aeronautics and Space Administration, Goddard Space Flight Center, Greenbelt, Maryland 20771, USA (2003)

14. Sickinger, C.: Verifikation entfaltbarer Composite-Booms für Gossamer-Raumfahrtsysteme; Shaker Verlag; ISBN 978-3-83228049-9 (2009)

15. Block, J., Straubel, M., Wiedemann, M.: Ultralight deployable booms for solar sails and other large Gossamer Structures in space; 60th International Astronautical Congress; Daejeon, Republic of Korea; 12-16, October (2009)

16. Herbeck, L., Eiden, M., Leipold, M., Sickinger, C., Unkenbold, W.: Development and test of deployable ultra-light weight cfrp boom for a solar sail; European Conference on Spacecraft Structures, Materials and Mechanical Testing; ESA-ESTEC, Noordwijk; 29.11.-1.12.00 (2000)

Publisher's Note Springer Nature remains neutral with regard to jurisdictional claims in published maps and institutional affiliations. 